\title{
Standounkt
}

Digitalisierung und Nachhaltigkeit

\section{Chancen und Sprengstoff}

\author{
Die Digitalisierung wird unser Leben auf eine sehr radikale Weise \\ verändern. Es ist daher wichtig, digitale Transformation nicht \\ nur kritisch zu diskutieren, sondern auch Leitplanken für diesen \\ gesellschaftlichen Wandel zu definieren - nach dem Motto: \\ Aufhalten geht nicht, aber Mitgestalten ist nötig. \\ Von Martin Oldeland
}

D er digitale Wandel rauscht mit hoher Geschwindigkeit, Dynamik, Komplexität und Euphorie über uns hinweg und verändert unser Leben auf eine sehr radikale Weise. Wenn wir dann noch die immensen Möglichkeiten der künstlichen Intelligenz berücksichtigen, werden sowohl die Geschwindigkeit als auch die möglichen Anwendungen und Auswirkungen noch um ein Vielfaches zunehmen. Der digitale Wandel ist eine logische Folge des Denkens, dass alles, was technisch möglich ist, auch gemacht oder zumindest ausprobiert werden sollte. Schöne neue Welt! Star Trek wird Realität.

Eine zentrale Frage ist, ob wir diese digitale Transformation in allen Bereichen unseres Lebens einfach so geschehen lassen sollen, ob wir die sich bereits abzeichnenden massiven Auswirkungen auch auf unser Gesellschaftssystem einfach ignorieren können. Was wird überwiegen: positive oder negative Auswirkungen? Es gilt nicht, nur den kritischen Diskurs zum Thema digitale Transformation zu führen, sondern auch Leitlinien und Leitplanken zu definieren, damit dieser Wandel gesellschaftspolitisch nicht aus dem Ruder läuft.

\section{Ökonomische Treiber ...}

Um die großen Herausforderungen unserer Zeit wie Klimawandel, Ressourcen, Energie, Biodiversität etc. erfolgreich meistern zu können, braucht es ein sehr viel stärkeres Maß an nachhaltiger Entwicklung. Die Vereinten Nationen haben mit den 17 Sustainable Deve- lopment Goals (SDG) einen Kompass verabschiedet, der die grundlegenden Problemfelder in den Mittelpunkt des gesellschaftlichen Handelns rückt. Nachhaltige Entwicklung bedeutet eben, neben den ökonomischen auch ökologische und soziale Aspekte sowie die kulturelle Dimension zu berücksichtigen.

Digitalisierung ist sehr stark getrieben von der ökonomischen Seite. Es geht um Effizienzgewinne, neue Technologien, neue Produkte, Kostensenkungen und ähnliche ökonomische Fragestellungen. Wie sieht es aber mit den ökologischen Folgen der Digitalisierung aus? Welche positiven und negativen Auswirkungen bringt sie mit sich?

Wir können zudem Rebound- oder Backfire-Effekte nicht ignorieren. Ein großes Thema ist der 3-D-Druck mit seinem Potenzial, Ressourcenverbräuche zu reduzieren. In manchen Anwendungsfällen, beispielsweise in der Medizin, ist 3-D-Druck sicherlich äußerst segensreich, bei der Herstellung von Ersatzteilen z. B. auch von Vorteil, aber in vielen anderen Fällen muss man aus gesamtökologischer Betrachtung die Frage stellen, ob alles Machbare auch sinnvoll ist.

\section{... und soziale Folgen}

Zur Nachhaltigkeit gehört auch die soziale Seite. Welche gesellschaftlichen Auswirkungen bringt die Digitalisierung also mit sich? Der digitale Wandel trifft zumindest bei uns auf eine deutlich ältere Gesellschaft, als dies bei anderen technologischen Entwicklungen zuvor der Fall war. Laut einer Studie sind allein in Deutschland bis zu 18 Millionen Arbeitsplätze durch den digitalen Wandel betroffen. Viele Tätigkeiten, Arbeitsplätze und Berufsfelder werden durch Maschinen und Software ersetzt.

Was passiert aber mit den freigesetzten Arbeitnehmern? Können wir diese wahrscheinlich eher älteren Menschen $\mathrm{zu}$ Digitalisierungsexperten umschulen und wird es entsprechende Arbeitsplätze für sie geben oder wird nicht vielleicht ein Großteil in die Arbeitslosigkeit oder prekäre Arbeitsverhältnisse abdriften? Führt dies langfristig für die Betroffenen zu einer noch früheren Altersarmut und werden wir durch die geringere Anzahl verbleibender Arbeitsplätze Probleme mit der Finanzierung der Sozialversicherungssysteme bekommen?

\section{Chancen durch soziale Innovation}

Aus meiner Sicht stecken in der noch recht jungen digitalen Transformation vielfältige Chancen, aber auch eine große Menge gesellschaftspolitischen Sprengstoffs. Wir werden die Themen Arbeit und Verteilung von Arbeit sowie Umgang mit persönlichen Daten sicherlich überdenken müssen. Die vielfältigen Diskussionen über eine mögliche Art eines Grundeinkommens sind ebenso notwendig wie die Frage, wie wir unsere Sozialversicherungssysteme aufrechterhalten können. Muss es nicht eine Art von wie auch immer gearteter Digitalisierungsabgabe geben, die genau diese notwendigen Finanzierungsbeiträge erbringt?

Wir brauchen also neben technischen Innovationen ganz dringend auch soziale Innovationen. Die rasante technologische Evolution braucht auch ein Pendant im Bereich der gesellschaftlichen Weiterentwicklung. Aufhalten geht nicht, aber Mitgestalten ist nötig.

AUTOR + KONTAKT Martin Oldeland ist Vorstand von B.A.U.M. e. V. B.A.U.M. e. V., Osterstraße 58, 20259 Hamburg. Tel.: +49 $40490711-00$ E-Mail: vorstand@baumev.de 